\title{
REVISTA BRASILEIRA DE
} AGRICULTURA RRIGADA

Revista Brasileira de Agricultura Irrigada v.13, nº.5, p. 3610 - 3618, 2019

ISSN 1982-7679 (On-line)

Fortaleza, CE, INOVAGRI - http://www.inovagri.org.br

DOI: $10.7127 /$ rbai.v13n5001119

Protocolo 1119.19 - 25/03/2020 Aprovado em 30/03/2020

\section{IDENTIFICAÇÃO DA INFLUÊNCIA DA PLUVIOMETRIA NO RENDIMENTO DO MILHO NO SEMIÁRIDO BRASILEIRO}

\author{
José Ricardo Ferreira Lopes ${ }^{1}$, Milena Pereira Dantas², Francisco Edson Paulo Ferreira ${ }^{3}$
}

\begin{abstract}
RESUMO
A produtividade de diversas culturas agrícolas é intensamente dependente da oferta pluviométrica, bem como de sua frequência e intensidade. O objetivo deste trabalho foi classificar a região semiárida brasileira em regiões homogêneas através da análise de agrupamento, levando-se em consideração a precipitação e a produtividade do milho, além de analisar a tendência temporal da precipitação por meio do teste de Mann-Kendall. Desse modo, pôde-se dividir a área de estudo em quatro grupos, evidenciando que o semiárido brasileiro é afetado pela alta variabilidade intrasazonal e interanual da precipitação que influencia diretamente no rendimento do milho. Os grupos 2 e 4 apresentaram uma tendência de aumento nos totais médios de produtividade do milho e tendência negativa na precipitação, isso pode ser explicado pela possível adoção da prática de irrigação, como também pelo o uso de variedades mais resistentes à seca, mecanização e manejo de solo. O milho é uma das culturas de maior importância econômica no semiárido, no entanto, os agricultores não conseguem explorar a potencialidade produtiva da cultura em virtude da alta variabilidade climática da chuva o que condiciona baixos rendimentos do grão.
\end{abstract}

Palavras-chave: tendências de chuva, produtividade, caatinga.

\section{REGIONALIZATION AND TRENDS PLUVIOMETRIC IN BRAZILIAN SEMI-ARID: INFLUENCES IN CORN YIELD}

\begin{abstract}
The productivity of various agricultural crops is strongly dependent on the rainfall supply, as well as its frequency and intensity. The objective of this work was to classify the Brazilian semiarid region in homogeneous regions through cluster analysis, taking into consideration the precipitation and corn yield, besides analysis the time trend of precipitation by means of the Mann-Kendall test. Thus, it

\footnotetext{
${ }^{1}$ Professor da Universidade do Estado de Minas Gerais, Doutorando em Meteorologia Aplicada, Universidade Federal de Viçosa, Viçosa, Minas Gerais, Brasil, e-mail: agrojrfl@gmail.com

${ }^{2}$ Doutoranda em Meteorologia, Instituto Nacional de Pesquisas Espaciais, Cachoeira Paulista, São Paulo, Brasil, e-mail: milena.dantas@gmail.com

${ }^{3}$ Doutorando em Meteorologia, Universidade Federal de Campina Grande, Campina Grande, Paraíba, Brasil, e-mail: edsonjua2009@gmail.com
} 
was possible divide study area in four groups, evidencing that the Brazilian semiarid is affected by the high intrasazonal and interannual variability of precipitation which directly influences in maize yield. The groups 2 and 4 show an crescent trend in maize average productivity total and negative trend in precipitation, this can be explained by the possible adoption of the irrigation practice, as also by the use of varieties that are more resistant to drought, mechanization and management of soil. Corn is one of the crops of greater economic importance in the semiarid, however, farmers fail to get explore the productive potential of culture due to the high climatic variability of rain which conditions low grain yield.

Keywords: rain trends, productivity, caatinga.

\section{INTRODUÇÃO}

O sistema agrícola do Semiárido brasileiro, de maneira geral, enfrenta dificuldades para atingir o seu máximo desenvolvimento produtivo. Isso se deve a problemas relacionados à irregularidade das chuvas (tanto espacialmente, como temporalmente), resultando em secas severas e prolongadas, assim como chuvas excessivas. Acrescido a isso, a maioria dos agricultores vive à margem da pobreza, possui terra insuficiente, a reserva de capital é insignificante, o capital humano é baixo, o nível de organização produtiva é rudimentar e os indicadores de progresso tecnológicos revelam regresso (MARENGO et al., 2016).

O milho Zea mays L., é uma planta da família Poaceae, originária da América Central, sendo cultivada em praticamente todas as regiões do mundo, nos hemisférios norte e sul, em climas úmidos e regiões secas. Em função de seu potencial produtivo, composição química e valor nutritivo, o milho constitui-se em um dos mais importantes cereais consumidos e é o mais produzido no mundo. Estudos que analisam a variabilidade climática em áreas de importância socioeconômica são de proeminente importância, sendo que por meio desses estudos é possível conhecer os problemas da região para, assim, tentar viabilizar um possível planejamento de políticas e ações no setor social, econômico e ambiental (LOPES et al., 2019).

Projeções indicam que o setor agrícola, por depender diretamente de temperatura e precipitação, será um dos mais afetados pelas mudanças do clima. Mesmo em países em desenvolvimento, como no caso do Brasil, espera-se que perdas de produtividade agrícola mesmo com todos os avanços tecnológicos possam chegar até $25 \%$ (NELSON et al., 2014).

Não obstante, por ser um país de dimensões continentais, esse valor pode ser muito distinto regionalmente. Soma-se a isso o fato de que diferentes tipos de agricultores sofrerão impactos distintos. Em especial, a agricultura familiar sofrerão os maiores impactos negativos. De acordo com Cunha et al. (2015), esses produtores são particularmente susceptíveis devido à sua localização geográfica (fortes déficits hidrológicos, altas temperaturas), baixos níveis de renda e desenvolvimento tecnológico, grande dependência da agricultura (predominantemente de sequeiro) e limitada capacidade adaptativa.

Vários estudos recentes abordaram a questão do efeito da variabilidade climática sobre as atividades e produtividade agrícola de agricultores familiares no Brasil (RAY et al., 2015; HERRERA et al., 2018) e como seu território semiárido é uma das regiões mais vulneráveis do país, em termos de impactos adversos esperados nas mudanças climáticas e vulnerabilidades sociais prevalentes, também ganha destaque na ciência global (BARBOSA e LAKSHMI KUMAR, 2016; GORI MAIA et al., 2018).

Diante do exposto objetivou-se classificar a região semiárida brasileira em regiões homogêneas levando em consideração a precipitação e a produtividade do milho, além de analisar possíveis tendências temporais da precipitação por meio do teste de Mann-Kendall. 


\section{MATERIAL E MÉTODOS}

O Semiárido brasileiro, em sua nova delimitação que entrou em vigor em 2017 (Figura 1), ocupa 1,12 milhão de $\mathrm{km}^{2}(13,25 \%$ da área do País), engloba 1.262 municípios e abriga aproximadamente 27,87 milhões de habitantes, ou seja, $13,42 \%$ da população de acordo com o censo de 2016.

Incluem os Estados do Ceará, Rio Grande do Norte, Paraíba, Pernambuco, Sudeste do Piauí, Oeste de Alagoas e Sergipe, dois municípios do Maranhão, região central da Bahia e uma faixa que se estende pela parte nordeste de Minas Gerais. Seu sistema agrícola, de maneira geral, enfrenta dificuldades para atingir o seu máximo desenvolvimento produtivo agrícola devido, principalmente ao déficit hídrico e as altas temperaturas, aumento dos eventos de veranicos, além de ser a região brasileira que será mais afetada pelas mudanças climáticas globais. O parâmetro de maior destaque dessa região é o clima, responsável pela variação dos outros elementos que compõem as paisagens, como a vegetação, predominante Caatinga, considerada como a mais rica floresta seca da América do Sul.

Como ocorre na maioria das florestas tropicais secas, a Caatinga também está exposta a uma ampla variedade de ameaças, mas a principal delas é provocada por atividades humanas na substituição da vegetação natural por agricultura ou pastagem e posterior abandono.

Com isso, a Caatinga se torna cada vez mais fragmentada e essas atividades não sustentáveis têm levado ao empobrecimento ambiental em larga escala.

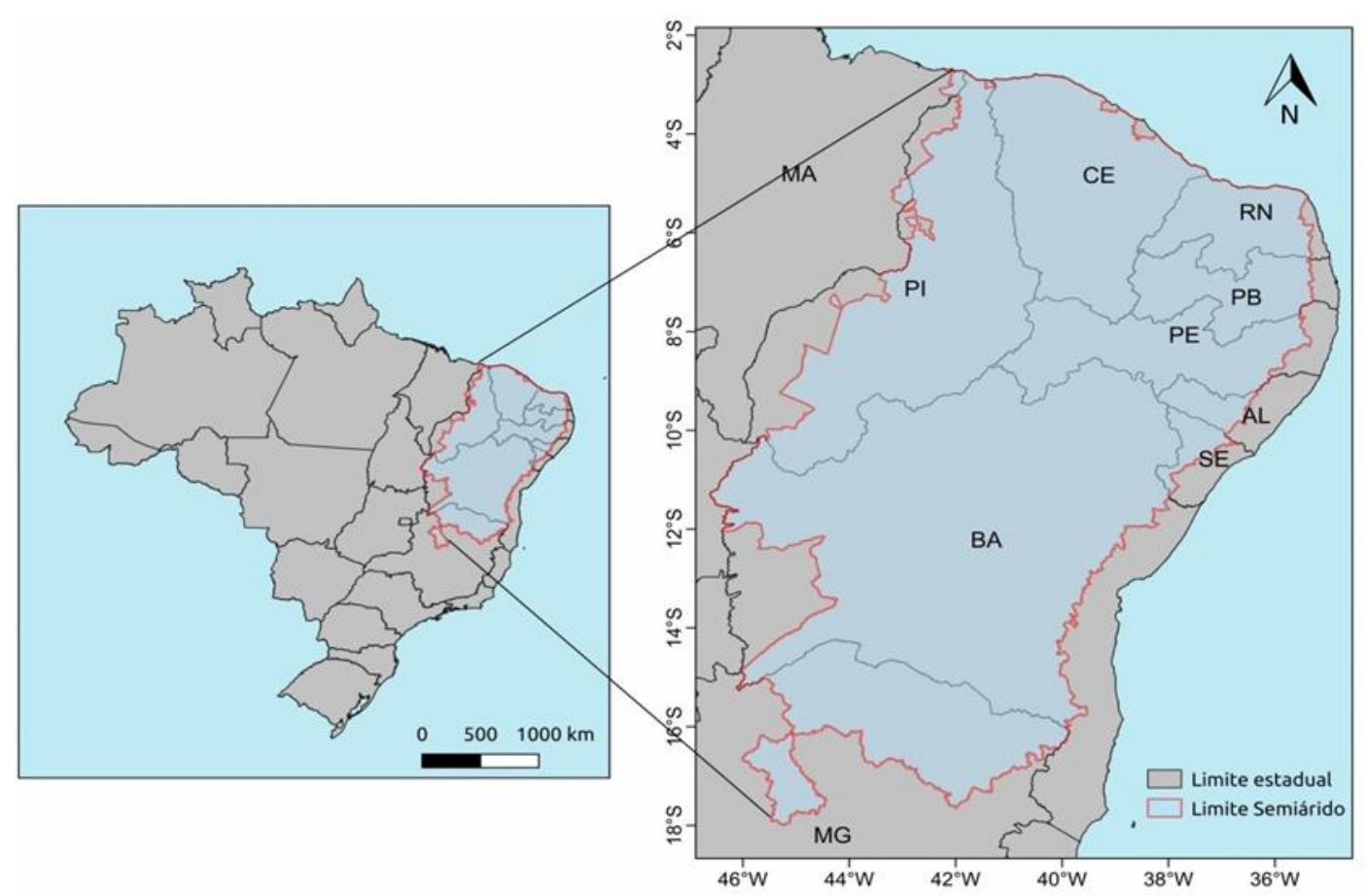

Figura 1. Mapa do Brasil com destaque a delimitação da região semiárida brasileira.

O conjunto de dados de precipitação pluvial utilizado nesta análise foi adquirido através do site <http://chg.geog.ucsb.edu/data/chirps/>, para o período de 1990 a 2014. Trata-se de estimativas de precipitação por satélite que fornecem informações com alta resolução espaço-temporal. Foi utilizada a versão mais recente do Climate Hazards Group InfraRed Precipitation with Station (CHIRPS v.2), produzida pela equipe conhecida como Climate Hazard Group InfraRed Precipitation (CHIRP), a qual fornece estimativas diárias de precipitação quase global $\left(50^{\circ} \mathrm{S}-50^{\circ} \mathrm{N} ; 180^{\circ}\right.$ $\left.\mathrm{E}-180^{\circ} \mathrm{W}\right)$. Este produto possui uma resolução espacial de $0,05^{\circ} \times 0,05^{\circ}(\sim 5,3 \mathrm{~km})$ 
e está disponível a partir do ano de 1981 até os dias atuais. Maiores detalhes da metodologia do banco de dados do CHIRPS podem ser encontrados em Funk et al. (2015).

As estimativas de precipitação por satélite são derivadas principalmente por sensores infravermelhos a bordo de satélites de órbita terrestre geossíncrona, ou seja, sua rotação acompanha exatamente a rotação da Terra, que podem ser adicionados ou não de sensores de microondas passivos e ativos a bordo de outros satélites. Outros produtos de estimativa de chuva combinam esses dois produtos, aproveitando assim a alta resolução temporal das plataformas de infravermelho e a melhor precisão na estimativa de precipitação de sensores (ABERA et al., 2016; MAGGIONI et al., 2016; KIMANI et al., 2017).

Paredes-Trejo et al. (2017) validaram os dados do CHIRPS para a região do nordeste brasileiro com várias métricas estatísticas, baseadas em comparação com dados de observações do pluviômetro de estação do Instituto Nacional de Meteorologia do Brasil (INMET), com o objetivo de avaliar o desempenho na estimativa da quantidade e da capacidade de detecção de chuva e apontaram alguns problemas de precisão, como a baixa capacidade de detecção de precipitação e, ao lidar com extremos, superestimando a precipitação no trimestre mais seco e subestimando os valores da chuva no trimestre mais chuvoso. Paredes-Trejo et al. (2019) validaram alguns produtos de precipitação por satélite para a região nordestina brasileira, incluindo o CHIRPS, com dados de observações em superfície provenientes da Agência Nacional de Águas (ANA), do INMET e do Departamento de Águas e Energia Elétrica, que foram ligadas usando um algoritmo de interpolação ponderada de distância inversa (XAVIER et al., 2016), mostrando a boa aplicabilidade do CHIRPS nessa região em comparação com demais produtos, assim como suas vantagens sobre as estações meteorológicas tradicionais, devido à sua baixa densidade.

Estes e outros estudos (MARIANO et al., 2018; COSTA et al., 2019) demonstram que o produto do CHIRPS v.2 é um bom conjunto de dados para o monitoramento da precipitação na região semiárida do Brasil e também pode contribuir para uma melhor compreensão da variabilidade espacial e temporal da pluviometria, como também da seca nessa região.

Entre os diferentes produtos agrícolas encontrados na região, as lavouras de arroz, feijão, mandioca e milho se destacam como os principais produtos cultivados pelos agricultores familiares, por possuírem características como o alto valor nutritivo e o baixo custo de produção. Os dados de produtividade do milho advêm do recenseamento obtido no Instituto Brasileiro de Geografia e Estatística (IBGE) e no Instituto de Pesquisa Econômica Aplicada (IPEA) por meio do Sistema de Recuperação Automática - SIDRA. Selecionou-se o período de 1981 a 2018. A aplicação das técnicas estatísticas deu-se a partir da combinação dos dados de rendimento do milho com os dados de precipitação pluvial.

Análise de Agrupamento (AA) objetiva classificar, de acordo com as variáveis, indivíduos semelhantes entre si em grupos, de modo que o grau de associação era forte entre membros do mesmo cluster e fraco entre os demais membros. A distância euclidiana é a medida mais frequentemente empregada quando todas as variáveis são quantitativas. A distância euclidiana é utilizada para calcular essas semelhanças que consiste na soma dos quadrados das diferenças, sem calcular a raiz quadrada. Empregou-se o método de agrupamento de Ward que forma grupos, minimizando a dissimilaridade, ou minimizando o total das somas de quadrados dentro de grupos, também conhecida como soma de quadrados dos desvios (SQD). Detalhes podem ser encontrados em Melo et al. (2017).

O teste de Mann-Kendall (MK) é um teste não paramétrico proposto por Mann (1945) e melhorado por Kendall (1975) que consiste em comparar cada valor da série temporal com os valores restantes, sempre em ordem sequencial, o que significa que não assume qualquer distribuição de prioridade 
dos dados, ou seja, deve permanecer sempre a mesma (série aleatória simples) e é, portanto, robusto em comparação a outros testes paramétricos. Verificou-se ser uma excelente ferramenta para a detecção de tendências em séries de tempo variável hidrológica. Detalhes podem ser encontrados em Lopes e Da Silva (2016).

\section{RESULTADOS E DISCUSSÃO}

$\mathrm{Na}$ Figura $2^{\mathrm{a}}$ expõe a distribuição espacial das tendências de precipitação obtidas pelo teste de MK para a região semiárida brasileira. É possível observar que a maior parte do território semiárido possui tendência pluviométrica negativa, variando de $-8,0$ até $-4,0 \mathrm{~mm} \mathrm{ano}^{-1}$. Os menores valores das tendências estão localizados no sudoeste da Bahia, na ordem de $-10 \mathrm{~mm}^{\mathrm{ano}}{ }^{-1}$ e há núcleos com valores positivos de tendência, variando de 6,0 a 10,0 $\mathrm{mm} \mathrm{ano}^{-1}$, na porção oeste da área de estudo (sul do Piauí e noroeste da
Bahia), no norte do Piauí, sul do Ceará e um na porção central entre Pernambuco e Paraíba. A probabilidade que essas tendências tanto de aumento quanto de diminuição de precipitação são exibidas pela Figura 2B, na qual podemos observar que a probabilidade das tendências de diminuição ocorrer são maiores que as tendências de aumento, o que pode levar a inferir que os totais pluviométricos anuais tendem a diminuir com o passar dos anos. Por exemplo, os núcleos que possuem maiores probabilidades ( $\mathrm{p}$-valor $>0,95)$ de ocorrer diminuição de chuva estão localizados no sudoeste e noroeste baiano, ou seja, a probabilidade de diminuição dos totais anuais médios pluviométricos é maior que $95 \%$ (Figura 5B).

Por outro lado a maior parte da região semiárida brasileira apresenta baixa probabilidade de ocorrência das tendências (pvalor $<0,30$ ), isso inclui as áreas onde há uma tendência de aumento na precipitação, ou seja, o aumento não possui significância estatística.
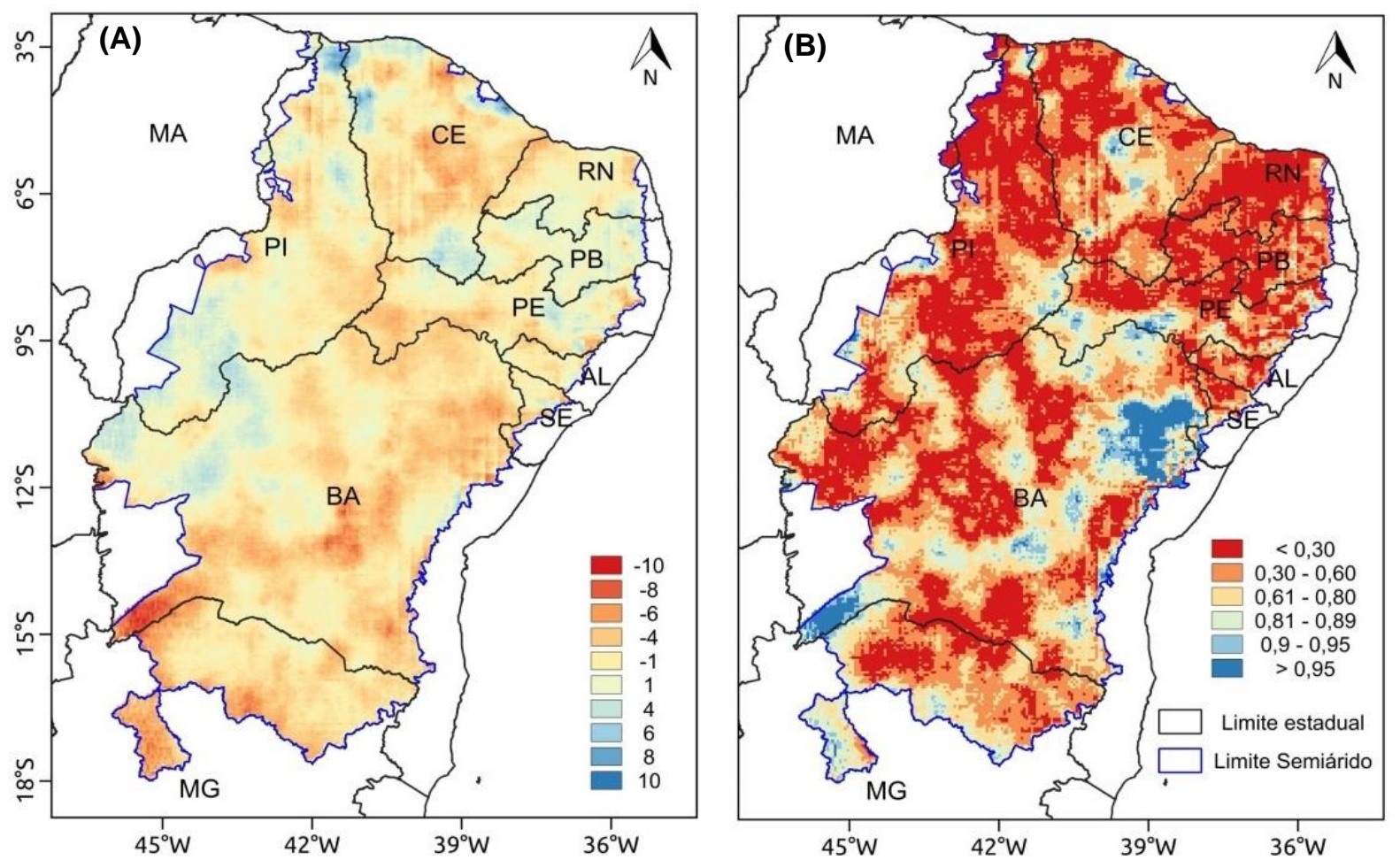

Figura 2. Distribuição espacial (A) e nível de significância (B) das tendências pluviométricas $\left(\mathrm{mm}^{\mathrm{ano}} \mathrm{o}^{-1}\right) \mathrm{obtidas}$ pelo teste de Mann-Kendall na região semiárida brasileira, no período de 1990 a 2014. Os pixels em azul escuro são aqueles que apresentam alterações estatisticamente significativas na pluviometria, de acordo com Teste de significância de T-Student $(\alpha=5 \%)$. 
A aplicação da AA dividiu a região semiárida brasileira em quatro clusters ou grupos (Figura 3), a saber: o grupo 1 situado na parte central da região compreendo os estados de Alagoas, Pernambuco, Paraíba, a maior parte do estado da Bahia e a porção central/sul do Piauí; o grupo 2 inserido nos estados do Ceará, Rio Grande do Norte, Sergipe e a parte norte do Piauí; o grupo 3 situados no norte de Minas Gerais (divisa com a Bahia) e norte da Bahia divisa com Pernambuco; e o grupo 4 posicionado em dois núcleos, um no oeste do estado do Piauí e o outro no centro/sul do estado da Bahia. Assim, esta análise conjunta das variáveis foi possível observar que este território apresenta alta variabilidade pluviométrica que influencia de forma direta na oscilação da produtividade do milho. Essa variabilidade espacial e interanual da chuva na região está associada a variações de padrões de Temperatura da Superfície do Mar (TSM) sobre os oceanos tropicais, o que pode interferir na atuação de sistemas atmosféricos pertencentes às várias escalas de tempo, como descrito em Ferreira et al. (2017).

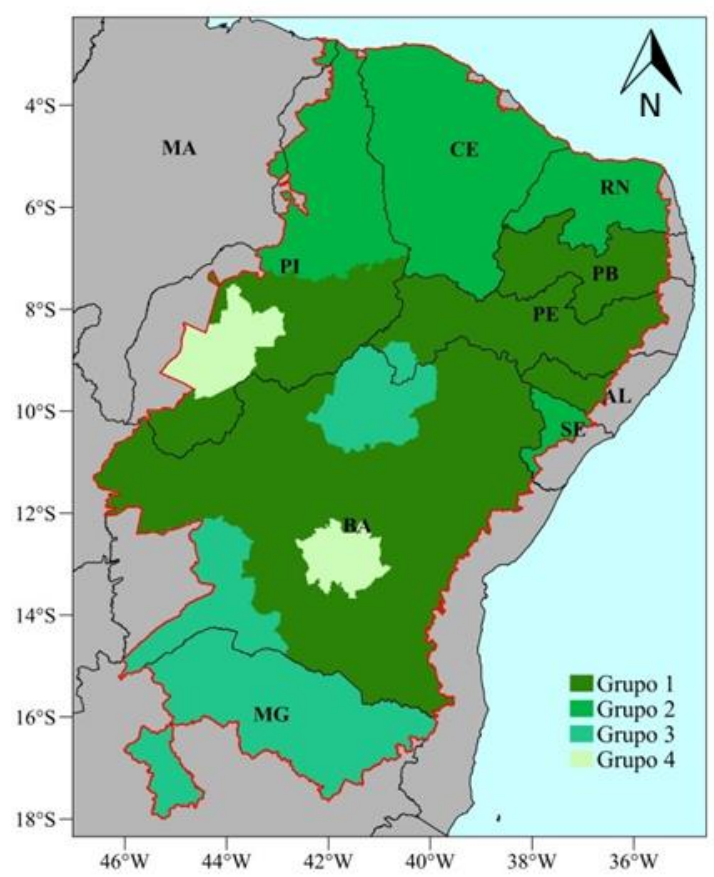

Figura 3. Regiões homogêneas na região semiárida brasileira.

Observar-se o clima seco no interior desta região pertence ao grupo (Figura 4A), onde as médias anuais precipitação para o período de estudo foram por volta de $500 \mathrm{~mm}$, apresentando uma tendência negativa, no valor de $-1,7 \mathrm{~mm}$ ano $^{-1}$. No entanto, a produtividade do milho para este grupo foi positiva, na ordem de 4,1 kg ha ano ${ }^{-1}$.

Ambas as tendências não apresentaram significância estatística. Diante do exposto pode-se observar nestes dois anos que a total anual de precipitação não implica na produtividade, é provável que isso ocorra devido a uma possível prática de irrigação ou pela grande variabilidade sazonal da chuva nesta área. O grupo 2 apresenta um clima intermediário, com maiores totais pluviométricos e maior variabilidade temporal comparando-se com o grupo 1, refletindo assim em maiores rendimentos do grão (Figura 4B). As tendências foram positivas: no valor de $5,8 \mathrm{~mm}$ ano $^{-1}$ para a pluviometria (sem significância estatística) e no valor de $18,5 \mathrm{~kg}$ ha ano $^{-1}$ para produtividade do milho. Um clima mais úmido, localizado principalmente na costa oeste e sul da região (grupos 3 e 4), com precipitação média anual em torno de $1.000 \mathrm{~mm}$, indicando assim, 
regiões mais e menos propícias ao cultivo desse grão.

$\mathrm{O}$ grupo 3 (Figura 4C) também apresentou tendências positivas, na pluviometria foi de $4,95 \mathrm{~mm} \mathrm{ano}^{-1}$ e no rendimento agrícola foi de $10,07 \mathrm{~kg}$ ha $\mathrm{ano}^{-1}$, as quais não foram estatisticamente
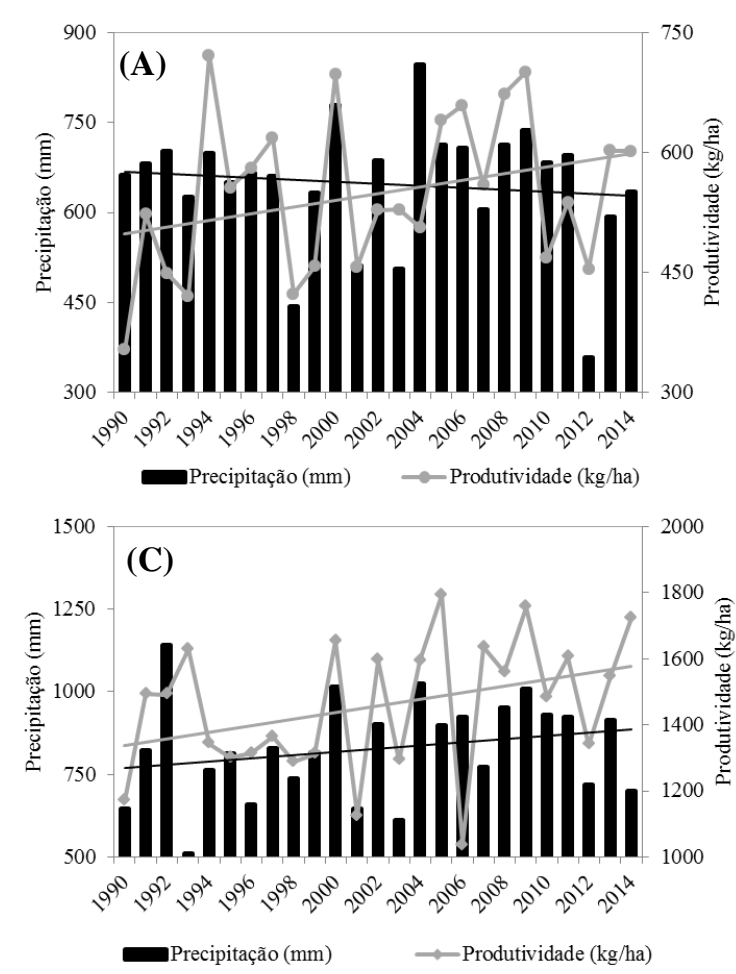

significativas. É nítido o crescimento da produtividade do milho temporalmente para o grupo 4 (Figura 4D) apresentou uma tendência negativa na ordem de $-2,86 \mathrm{~mm} \mathrm{ano}{ }^{-1}$, sem significância estatística e uma tendência positiva de produtividade do milho de 237,84 $\mathrm{kg}$ ha $\mathrm{ano}^{-1}$ para nível de significância de $5 \%$.
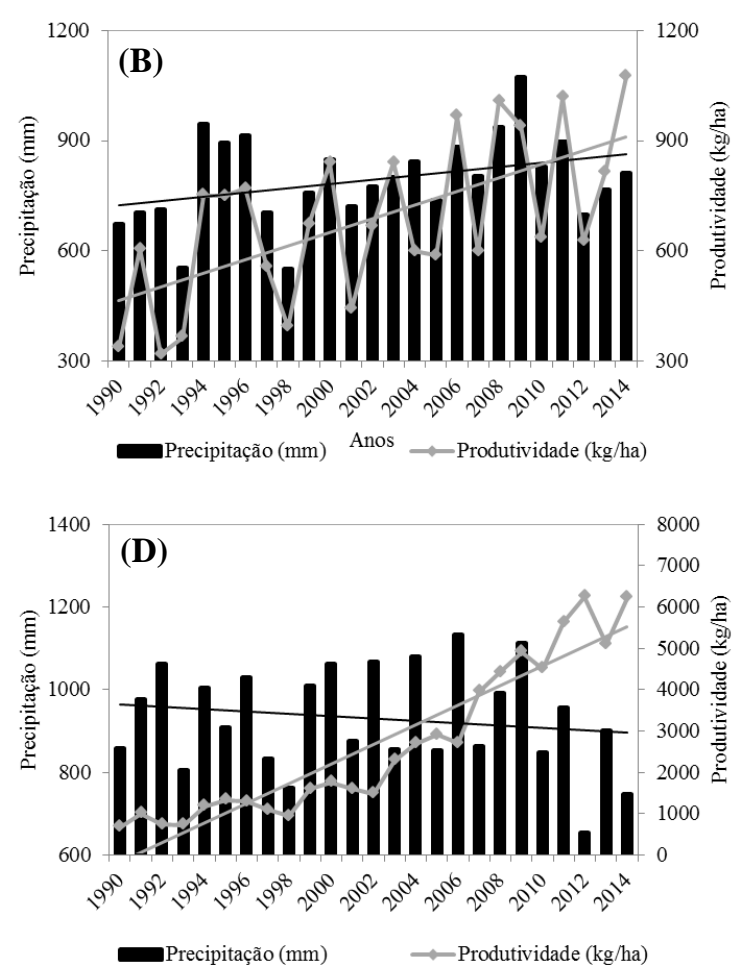

Figura 4. Variabilidade anual dos totais médios pluviométricos e da produtividade do milho do grupo 1 (A), grupo 2 (B), grupo 3 (C) e grupo 4 (D) na região semiárida brasileira.

Estudos como o de Bustos et al. (2013) identificaram que a partir dos anos 2000 que houve uma introdução de novas tecnologias nas principais safras do país (milho geneticamente modificado, por exemplo), afetando significativamente os totais da produtividade do milho.

Analisando os grupos 2 e 4 identifica-se uma tendência de aumento nos totais médios de produtividade do milho, e tendência negativa na precipitação. Isso pode ser explicado em parte pelas mudanças na estrutura produtiva.

De modo geral, o padrão de variabilidade da chuva e da produtividade do milho é, parcialmente, interdependente, ou seja, há áreas no semiárido brasileiro que são mais/menos atingidas negativamente por eventos de seca. Isso se deve as tecnologias adotadas no sistema de produção da cultura, destacando-se o melhoramento genético, variedades mais resistentes à seca, manejo de solo e o emprego da mecanização em diversos processos, como apontado por Lopes et al. (2019).

\section{CONCLUSÕES}

Evidenciou-se que em apenas $10 \%$ do território pertencente ao semiárido brasileiro a produtividade do milho foi superior a $1000 \mathrm{~kg}$ ha $^{-1}$ durante o período de 1990 a 2014, a partir disto, pode-se concluir que o cultivo predominante é o de sequeiro. Foi possível identificar e agrupar quatro regiões 
homogêneas. Sendo que os grupos 2 e 4 apresentaram uma tendência de aumento nos totais médios de produtividade do milho, e tendência negativa na precipitação.

O milho é uma das culturas de maior importância econômica no semiárido, no entanto, os agricultores não conseguem explorar a potencialidade produtiva da cultura em virtude da alta variabilidade climática da chuva o que condiciona baixos rendimentos do grão.

\section{REFERÊNCIAS BIBLIOGRÁFICAS}

ABERA, W.; BROCCA, L.; RIGON, R. Comparative evaluation of different satellite rainfall estimation products and bias correction in the Upper Blue Nile (UBN) basin. Atmospheric Research, v. 178-179, p. 471-483,

DOI: https://doi.org/10.1016/j.atmosres.2016. 04.017

BARBOSA, H. A.; LAKSHMI KUMAR, T. $\mathrm{V}$. Influence of rainfall variability on the vegetation dynamics over Northeastern Brazil. Journal of Arid Environments, v. 124, p. 377-387, 2016.

BUSTOS, P.; CAPRETTINI, B.; PONTICELLI, J. Agricultural Productivity and Structural Transformation: evidence from Brazil. Economics Working Papers 1403, Department of Economics and Business, Universitat Pompeu Fabra, ago. 2013.

CHEN, Y.; GUAN, Y.; SHAO, G.; ZHANG, D. Investigating Trends in Streamflow and Precipitation in Huangfuchuan Basin with Wavelet Analysis and the Mann-Kendall Test. Water, v. 8, n. 3, p. 77, 2016.

COSTA, J. C.; PEREIRA, G.; SIQUEIRA, M. E.; CARDOZO, F. S.; SILVA, V. V. Validação dos dados de precipitação estimados pelo CHIRPS para o Brasil. Revista Brasileira de Climatologia, v. 24, p. 228-243, 2019. DOI: http://dx.doi.org/10.5380/abclima.v24i0.60237
FERREIRA， P. S.; GOMES, V. P.; GALVÍNCIO, J. D.; SANTOS, A. M.; SOUZA, W. M. Avaliação da tendência espaço-temporal da precipitação pluviométrica em uma região semiárida do estado de Pernambuco. Revista Brasileira de Climatologia, v. 21, p. 113-134, jul./dez. 2017.

FUNK, C.; PETERSON, P.; LANDSFELD, M.; PEDREROS, D.; VERDIN, J.; SHUKLA, S.; HUSAK. G.; ROWLAND, J.; HARRISON, L.; HOELL, A.; MICHAELSEN, J. The climate hazards infrared precipitation with stations - a new environmental record for monitoring extremes. Scientific Data, v. 2, 2015. DOI: http://dx.doi.org/10.1038/sdata.2015.66

GORI MAIA, A.; CESANO, D.; MIYAMOTO, B. C.B.; EUSEBIO, G. S.; SILVA, P. A. O. Climate change and farmlevel adaptation: the Brazilian Sertão. International Journal of Climate Change Strategies and Management, v. 2018, p. 123, 2018.

KENDALL, M. G. Rank correlation measures. Charles Griffin: London, U.K, p.220, 1975

KIMANI, M .W.; HOEDJES, J .C .B.; SU, Z. An assessment of satellite-derived rainfall products relative to ground observations over East Africa. Remote Sensing, v. 9, p. 430. 2017. DOI: https://doi.org/10.3390/rs9050430

LOPES, J. R. F.; Da SILVA, D. F. Tendências e distribuição espacial de variáveis climáticas no estado do Ceará. Ambiência Guarapuava (PR) v.12 n.1 p. 127 - 145 Jan./Abr. 2016.

LOPES, J. R. F.; DANTAS, M. P.; FERREIRA, F. E. P. Variabilidade da precipitação pluvial e produtividade do milho no semiárido brasileiro através da análise multivariada. Revista Nativa, v. 7, n. 1, p. 7783, jan./fev. 2019.

MAGGIONI, V.; SAPIANO, M. R. P.; ADLER, R. F. Estimating Uncertainties in 
High-Resolution Satellite Precipitation Products: Systematic or Random Error? Journal Hydrometeorology, v. 17, p. 11191129, 2016.2 DOI: https://doi.org/10.1175/JHM-D-15-0094.1 MANN, H. B. Econometrical. The econometric society, v.13, n.3, p.245-259, 1945.

MARENGO, J. A.; TORRES, R. R.; ALVES, L. M. Drought in Northeast Brazil - past, present and future. Theoretical and Applied Climatology. 20: 1-12, 2016.

MARIANO, D. A.; SANTOS, C. A. C.; WARDLOW, B. D.; ANDERSON, M. C.; SCHILTMEYER, A. V.; TADESSE, T.; SVOBODA, M. D. Use of remote sensing indicators to assess effects of drought and human-induced land degradation on ecosystem health in Northeastern Brazil. Remote Sensing of Environment, v. 213, p. 129-143, 2018.2 DOI: https://doi.org/10.1016/j.rse.2018.04.048

MELO, V. S.; Do Ó, K. D. S.; DANTAS, M. P.; MEDEIROS, R. M. Estudo da precipitação do semiárido baiano usando análises de agrupamento e distribuição de probabilidade. Revista Brasileira de Agrotecnologia, v. 7, p. 11-19, n. 2017.

NELSON, G. C. VALINB, H.; SANDSC, R. D.; HAVLÍKB, P.; AHAMMADD, H.; DERYNGE, D.; ELLIOTTF, J.; FUJIMORIH, S.; HASEGAWAH, T.; HEYHOED, E.; KYLEI, P.; VON LAMPEJ, M.; LOTZE-
CAMPEN, H.; D'CROZA, D.; VAN MEIJLL, H.; VAN DER MENSBRUGGHEM, D.; MÜLLER, C.; POPP, A.; ROBERTSONA, R.; ROBINSONA, S.; SCHMIDN, E.; SCHMITZK, C.; TABEAUL, A.; WILLENBOCKELO, D. Climate change effects on agriculture: Economic responses to biophysical shocks. Proceedings of the National Academy of Sciences, v. 111, n. 9, p. 3274-3279, 2014.

PAREDES-TREJO F. J.; BARBOSA H. A; LAKSHMI KUMAR T. V. Validating CHIRPS-based satellite precipitation estimates in Northeast Brazil. Journal of Arid Environments, v. 139, p. 26-40, 2017. DOI: https://doi.org/10.1016/j.jaridenv.2016.12.009

PAREDES-TREJO, F.; BARBOSA, H. A.; SANTOS, C. A. C. Evaluation of the Performance of SM2RAIN-Derived Rainfall Products over Brazil. Remote Sensing, v. 11, p. $1113, \quad 2019.2$ DOI: https://doi.org/10.3390/rs11091113

RAY, D. K.; GERBER, J. S.; MACDONALD, G. K.; WEST, P. C. Climate variation explains a third of global crop yield variability. Nature Communication., 6, p. 5989, 2015. DOI: https://doi.org/10.1038/ncomms6989

XAVIER, A. C.; KING, C. W.; SCANLON, B. R. Daily gridded meteorological variables in Brazil (1980-2013). International Journal of Climatology, v. 36, p. 2644-2659, 2015. DOI: https://doi.org/10.1002/joc.4518 